\title{
THE CONTINUOUS WAVELET TRANSFORM ON CONIC SECTIONS
}

\author{
Jean-Pierre Antoine* \\ Institut de Physique Théorique (FYMA), Université catholique de Louvain, \\ B-1348 Louvain-la-Neuve, Belgium \\ I. Bogdanova ${ }^{\dagger}$ \\ University of Neuchâtel, Institute of Microtechnology, Pattern Recognition Lab. \\ Rue A.-L. Breguet 2, CH-2000 Neuchâtel, Switzerland \\ P. Vandergheynst ${ }^{\ddagger}$ \\ Signal Processing Institute, Swiss Federal Institute of Technology, EPFL \\ Station 11, CH-1015 Lausanne, Switzerland \\ Received (Day Month Year) \\ Revised (Day Month Year) \\ Communicated by (xxxxxxxxxx)
}

\begin{abstract}
We review the coherent state or group-theoretical construction of the continuous wavelet transform (CWT) on the two-sphere. Next we describe the construction of a CWT on the upper sheet of a two-sheeted hyperboloid, emphasizing the similarities between the two cases.. Finally we give some indications on the CWT on a paraboloid and we introduce a unified approach to the CWT on conic sections.
\end{abstract}

Keywords: continuous wavelet transform, conic sections, two-sphere, two-sheeted hyperboloid, paraboloid

AMS Subject Classification: 42C15, 42C40, 65T60

\section{Introduction: In the footsteps of Apollonius}

It is well-known that many processes in physics, astronomy and medicine yield data on spherical manifolds. But there are situations where data live on other nonEuclidean manifolds, for instance, in cosmology (an open expanding model of the universe has the geometry of two-sheeted hyperboloid) or in optics (in catadioptric image processing, where a sensor overlooks a hyperbolic or parabolic mirror). ${ }^{12,18}$ In this catadioptric procedure, optical data may be processed directly on the mirror,

\footnotetext{
*Antoine@fyma.ucl.ac.be

†iva.bogdanova@unine.ch

${ }^{\ddagger}$ pierre.vandergheynst@epfl.ch
} 
thus on a two-sheeted hyperboloid or a paraboloid. ${ }^{12}$ So one needs suitable analysis tools for data living on such non-Euclidean manifolds. This suggests to adapt the continuous wavelet transform (CWT) to this situation.

Actually, those three manifolds constitute the so-called conic sections, generated by intersecting a double cone by a plane. This was a major discovery due to Apollonius of Perga (c. 262 BCc. 190 BC), a Greek geometer and astronomer of the Alexandrian school (incidentally, it was Apollonius who gave the ellipse, the parabola, and the hyperbola the names by which we know them ${ }^{42}$ ). Thus one should aim at designing a unified CWT formalism for all conic sections at once.

This paper is organized as follows. First we shall review very briefly the known construction of the continuous wavelet transform (CWT) on the two-sphere. Next we turn to the corresponding construction on the upper sheet of two-sheeted hyperboloid. As will be seen, the two cases are largely parallel to each other. Then, after a few remarks on the case of the paraboloid, we give some indications on a unified approach to the CWT on conic sections.

\section{The CWT on the two-sphere}

Let us begin with the two-sphere $S^{2}$. Fourier analysis on $S^{2}$ is standard, but cumbersome, since it amounts to work with expansions in spherical harmonics. The latter, denoted $\left\{Y_{l}^{m}\right\}$, constitute an orthonormal basis of $L^{2}\left(S^{2}, d \mu\right)$, so that any function $f \in L^{2}\left(S^{2}, d \mu\right)$ may be expanded as

$$
\begin{aligned}
f(\omega) & =\sum_{l \in \mathbb{N}} \sum_{|m| \leqslant l} \widehat{f}(l, m) Y_{l}^{m}(\omega), \\
\widehat{f}(l, m) & =\left\langle Y_{l}^{m} \mid f\right\rangle=\int_{S^{2}} d \mu(\omega) \overline{Y_{l}^{m}(\omega)} f(\omega),
\end{aligned}
$$

where $\omega=(\theta, \varphi) \in S^{2}, \theta \in[0, \pi]$ is the latitude angle, $\varphi \in[0,2 \pi)$ is the longitude angle, and $d \mu=\sin \theta d \theta d \varphi$ is the $\mathrm{SO}(3)$-invariant measure on $S^{2}$.

The problem is that, since $Y_{l}^{m}$ are not localized at all on the sphere, Fourier analysis is global, whereas experience tells us that a local analysis is highly desirable. Actually, there are specific combinations of spherical harmonics which are well localized (the so-called spherical harmonics kernels ${ }^{31}$ ), but their use entails losing the simplicity of an orthonormal basis.

Thus it is not surprising that alternative solutions have been proposed. Besides Gabor analysis on the tangent bundle, ${ }^{38}$ one finds in the literature several approaches related to the continuous wavelet transform and corresponding frames. For instance, Freeden et al. ${ }^{15,17}$ combine wavelets and spherical harmonics, defining a transformation on $S^{2}$ using a special dilation operator defined on the Fourier domain. Mhaskar et al..$^{30}$ introduce polynomial spherical frames where the order of the polynomials plays the role of the dilation. The drawbacks of these methods is that they focus on the frequential aspect of the transformations. In consequence, the spatial localization of these wavelets is either not guaranteed or precisely con- 
trolled. On the other hand, these kernel methods lead to the rich field of spherical approximations ${ }^{5,16}$ and prove very useful in the geosciences.

Bülow ${ }^{13}$ did succeed in getting good localization properties by using the evolution of a spherical Gaussian governed by the heat equation on $S^{2}$. Then he gets a set of wavelet filters by differentiation of this Gaussian. However, this approach is restricted to the Gaussian function and thus it not as general as the one based on a stereographic dilation applied to an arbitrary admissible wavelet on $S^{2}$, that we shall describe in the sequel.

We ought also to mention the work of Holschneider et al. on Poisson multipole wavelets, ${ }^{14,25,26}$ which are presented as a substitute to spherical harmonics kernels. The resulting frames prove indeed very efficient in geophysics, in particular for the representation of the Earth's magnetic and gravity fields.

Discrete wavelets on the sphere have also been designed, using an $S^{2}$ multiresolution analysis. For instance, Haar wavelets on a triangulation of $S^{2}$ and refined with the lifting scheme were introduced by Schröder and Sweldens. ${ }^{36}$ This approach has become a common tool in computer graphics. We may also mention $C^{1}$ wavelets constructed by a factorization of the refinement matrices; ${ }^{39}$ or wavelets obtained by radial projection from a polyhedron inscribed in the sphere, typically locally supported spline wavelets on spherical triangulations ${ }^{32,34}$ (actually this construction extends to sphere-like surfaces, i.e., continuous deformations of a sphere ${ }^{33}$ ). It is interesting to note that the authors of Refs. 14, 25 also use a radial projection from a polyhedron contained in the sphere, namely a cube, but only for defining a discretization of the positions on the sphere - and then they construct frames, not orthonormal bases. References to the (vast) literature on discrete spherical wavelets may be found in Refs. 32 and 39 for earlier work and in Ref. 29 for recent work.

However, various problems plague most of those constructions, such as an inadequate notion of dilation, the lack of wavelet localization, the excessive rigidity of the wavelets obtained, the lack of directionality, etc. In this respect, the continuous wavelet transform (CWT) has many advantages: locality is controlled by dilation, the wavelets are easily transported around the sphere by rotations from $\mathrm{SO}(3)$, efficient algorithms are available. Holschneider ${ }^{24}$ was the first to build a genuine spherical CWT, but his construction involves several assumptions and lacks a geometrical feeling. In particular, it contains a parameter that has to be interpreted as a dilation parameter, but whose geometrical meaning is unclear. A satisfactory solution (including a group-theoretical derivation of all the assumptions of Holschneider ${ }^{24}$ ) was obtained in a series of papers from our groups ${ }^{3,4,5,9}$ that yield a rigorous and efficient spherical CWT. A further simplification was obtained later by invoking conformal arguments. ${ }^{40}$ In the sequel of this section, we shall give a rapid overview of the series of works mentioned above, following mostly Ref. 7. As a general reference on 2-D wavelets, we use our recent monograph. ${ }^{6}$ For group-theoretical notions, we refer to standard textbooks, such as those of Barut-Raczka ${ }^{8}$ or Gilmore. ${ }^{19}$ 


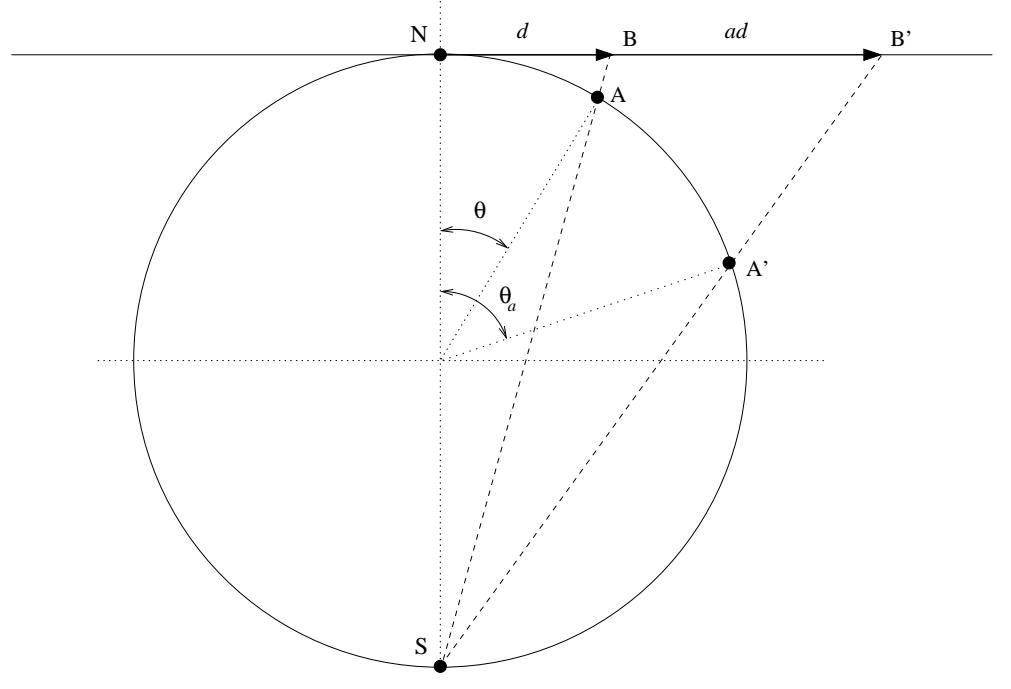

Fig. 1. Visual meaning of the stereographic dilation on $S^{2}$.

\subsection{The spherical CWT: sketching the problem}

As it is well-known, the design of a CWT on a given manifold $X$ starts by identifying the operations one wants to perform on the finite energy signals living on $X$, that is, functions in $L^{2}(X, d \nu)$, where $\nu$ is a suitable measure on $X$. Next one realizes these operations by unitary operators on $L^{2}(X, d \nu)$ and one looks for a possible group-theoretical derivation.

In the case of the two-sphere $S^{2}$, the required transformations are of two types: (i) motions, which are realized by rotations $\varrho \in \mathrm{SO}(3)$, and (ii) dilations of some sort by a scale factor $a \in \mathbb{R}_{+}^{*}$. The problem is how to define properly the dilation on the sphere $S^{2}$ itself.

A possible solution is to use a (radial) stereographic dilation on $S^{2}$, which is obtained in three steps (Figure 1): (i) given a point $\mathrm{A} \in S^{2}$, different from the South Pole $S$, project it stereographically to the point $B$ in the plane tangent to the sphere at the North Pole N; (ii) dilate B radially in the usual way to B'; and (iii) project back $B^{\prime}$ to the sphere, which yields $A^{\prime}$. The map $A \mapsto A^{\prime}$ is the required spherical dilation around $\mathrm{N}$. In order to dilate around any other point $\mathrm{C}$, just bring it to $\mathrm{N}$ by a rotation $\varrho \in \mathrm{SO}(3)$, dilate as above, and go back to $\mathrm{C}$ by the inverse rotation $\varrho^{-1}$.

The operations just defined have a natural realization by unitary operators in $L^{2}\left(S^{2}, d \mu\right)$ :

$$
\begin{aligned}
& \text {. rotation } R_{\varrho}:\left(R_{\varrho} f\right)(\omega)=f\left(\varrho^{-1} \omega\right), \varrho \in \mathrm{SO}(3), \\
& \text {. dilation } D_{a}:\left(D_{a} f\right)(\omega)=\lambda(a, \theta)^{1 / 2} f\left(\omega_{1 / a}\right), a \in \mathbb{R}_{+}^{*} .
\end{aligned}
$$


In these relations, $\omega_{a}=\left(\theta_{a}, \varphi\right), \theta_{a}$ is defined by $\tan \frac{\theta_{a}}{2}=a \tan \frac{\theta}{2}$ for $a>0$ and the normalization factor $\lambda(a, \theta)^{1 / 2}$ (called cocycle or Radon-Nikodym derivative) is needed for compensating the noninvariance of the measure $\mu$ under dilation. Explicitly, this factor is given by

$$
\lambda(a, \theta)=\frac{4 a^{2}}{\left[\left(a^{2}-1\right) \cos \theta+\left(a^{2}+1\right)\right]^{2}} .
$$

As we shall see below, one may derive a CWT from these ingredients, following the general coherent state formalism, ${ }^{1}$ as was the case for the 1-D and the 2-D plane CWT. But one may ask also whether this transformation is unique, as in the plane cases.

\subsection{The group-theoretical method}

According to the general scheme ${ }^{6}$ a possible way of solving the problem is to use the coherent state formalism relying on square integrable representations of a suitable transformation group. ${ }^{1}$ Thus we start from the affine transformations on $S^{2}$, which consists of rotations and dilations. These two types of operations do not commute, yet the only extension of $\mathrm{SO}(3)$ by $\mathbb{R}_{*}^{+}$is their direct product. In order to evade this contradiction, one embeds the two factors into the Lorentz group $\mathrm{SO}_{o}(3,1)$, by the so-called Iwasawa decomposition: ${ }^{19,22}$

$$
\mathrm{SO}_{o}(3,1)=\mathrm{SO}(3) \cdot A \cdot N,
$$

where $\mathrm{SO}(3)$ is the maximal compact subgroup of $\mathrm{SO}_{o}(3,1), A \simeq \mathrm{SO}_{o}(1,1) \simeq \mathbb{R} \simeq$ $\mathbb{R}_{*}^{+}$(boosts in the $z$-direction) and the nilpotent factor $N$ is here abelian and twodimensional, so that $N \simeq \mathbb{C}$. This procedure is justified by the fact that the Lorentz group $\mathrm{SO}_{o}(3,1)$ is the conformal group both for the sphere $S^{2}$ and for the tangent plane $\mathbb{R}^{2}$.

Next we have to compute the action of the Lorentz group on the sphere. The stability subgroup of the North Pole is $P=\mathrm{SO}_{z}(2) \cdot A \cdot N$. Thus $S^{2} \simeq \mathrm{SO}_{o}(3,1) / P \simeq$ $\mathrm{SO}(3) / \mathrm{SO}(2)$, so that $\mathrm{SO}_{o}(3,1)$ acts transitively on $S^{2}$. Then an explicit computation with help of the Iwasawa decomposition (2.6) shows that the pure dilation by $a$, realized as a Lorentz boost along the $z$-axis, coincides with the stereographic dilation (2.4).

Going over to the Hilbert space, we find that the Lorentz group $\mathrm{SO}_{o}(3,1)$ has a natural unitary irreducible representation (UIR) in $L^{2}\left(S^{2}, d \mu\right)$, namely, ${ }^{37}$

$$
[U(g) f](\omega)=\lambda(g, \omega)^{1 / 2} f\left(g^{-1} \omega\right), \quad \text { for } g \in \mathrm{SO}_{o}(3,1), f \in L^{2}\left(S^{2}, d \mu\right),
$$

where $\lambda(g, \omega)=\lambda(a, \theta)$ is the Radon-Nikodym derivative given in (2.5).

Thus the parameter space of spherical wavelets is the homogeneous space $X=$ $\mathrm{SO}_{o}(3,1) / N \simeq \mathrm{SO}(3) \cdot \mathbb{R}_{*}^{+}$, which is not a subgroup of $\mathrm{SO}_{o}(3,1)$. In order to apply the general formalism, we must introduce a section $\sigma: X \rightarrow \mathrm{SO}_{o}(3,1)$ and consider the 
reduced representation $U(\sigma(\varrho, a))$. Choosing the natural (Iwasawa) section $\sigma(\varrho, a)=$ $\varrho a, \varrho \in \mathrm{SO}(3), a \in A$, we obtain

$$
U(\sigma(\varrho, a))=U(\varrho a)=U(\varrho) U(a)=R_{\varrho} D_{a},
$$

exactly as before, in (2.3)-(2.4).

It turns out that the representation (2.8) has all the properties that are required to generate a useful CWT. First of all, it is square integrable on the quotient manifold $X=\mathrm{SO}_{o}(3,1) / N \simeq \mathrm{SO}(3) \cdot \mathbb{R}_{*}^{+}$. For simplicity, we shall identify these two isomorphic manifolds.

Proposition 2.1. The UIR (2.7) is square integrable on $X$, that is, there exist nonzero (admissible) vectors $\psi \in L^{2}\left(S^{2}, d \mu\right)$ such that

$$
\int_{0}^{\infty} \frac{d a}{a^{3}} \int_{\mathrm{SO}(3)} d \varrho|\langle U(\sigma(\varrho, a)) \psi \mid \phi\rangle|^{2}:=\left\langle\phi \mid A_{\psi} \phi\right\rangle<\infty, \text { for all } \phi \in L^{2}\left(S^{2}, d \mu\right) .
$$

Here de is the left Haar measure on $\mathrm{SO}(3)$.

The resolution operator (also called frame operator) $A_{\psi}$ is diagonal in Fourier space (i.e., it is a Fourier multiplier):

$$
\widehat{A_{\psi} f}(l, m)=G_{\psi}(l) \widehat{f}(l, m),
$$

where

$$
G_{\psi}(l)=\frac{8 \pi^{2}}{2 l+1} \sum_{|m| \leqslant l} \int_{0}^{\infty} \frac{d a}{a^{3}}\left|\widehat{\psi}_{a}(l, m)\right|^{2}, \quad \text { for all } l \in \mathbb{N},
$$

and $\widehat{\psi}_{a}(l, m)=\left\langle Y_{l}^{m} \mid \psi_{a}\right\rangle$ is the Fourier coefficient of $\psi_{a}=D_{a} \psi$.

Next, we have an exact admissibility condition on the wavelets (this condition was also derived by Holschneider in a somewhat ad hoc way). ${ }^{24}$

Proposition 2.2. An admissible wavelet is a function $\psi \in L^{2}\left(S^{2}, d \mu\right)$ for which there exists a positive constant $c<\infty$ such that

$$
G_{\psi}(l) \leqslant c, \text { for all } l \in \mathbb{N} .
$$

Equivalently, the function $\psi \in L^{2}\left(S^{2}, d \mu\right)$ is an admissible wavelet if and only if the resolution operator $A_{\psi}$ is bounded and invertible.

In addition, any admissible spherical wavelet $\psi$ such that $\int_{0}^{2 \pi} d \varphi \psi(\theta, \varphi) \not \equiv$ 0 , in particular, any nonzero axisymmetric wavelet, generates a continuous frame $\left\{\psi_{a, \varrho}:=R_{\varrho} D_{a} \psi: a>0, \varrho \in \mathrm{SO}(3)\right\}$, i.e., the operators $A_{\psi}$ and $A_{\psi}^{-1}$ are both bounded. These frames, however, are presumably nontight.

A simple example of admissible spherical wavelet is the Difference of Gaussians spherical wavelet (SDOG), obtained by transferring to the sphere the usual plane DOG wavelet. More precisely, for $\phi(\theta, \varphi)=\exp \left(-\tan ^{2}\left(\frac{\theta}{2}\right)\right)$, the SDOG wavelet is defined as

$$
\psi_{G}^{(\alpha)}(\theta, \varphi)=\phi(\theta, \varphi)-\frac{1}{\alpha}\left[D_{\alpha} \phi\right](\theta, \varphi), \quad \text { for } \alpha>0
$$


Using the previous results, we may now introduce the spherical CWT.

Definition 2.3. Given the admissible wavelet $\psi$, the spherical CWT of a function $f \in L^{2}\left(S^{2}, d \mu\right)$ with respect to $\psi$ is defined as

$$
W_{f}(\varrho, a):=\left\langle\psi_{a, \varrho} \mid f\right\rangle=\int_{S^{2}} d \mu(\omega) \overline{\left[R_{\varrho} D_{a} \psi\right](\omega)} f(\omega), \varrho \in S O(3), a>0 .
$$

According to the general coherent state formalism, there is a reconstruction formula. For any $f \in L^{2}\left(S^{2}, d \mu\right)$ and $\psi$ an admissible wavelet such that $\int_{0}^{2 \pi} d \varphi \psi(\theta, \varphi) \not \equiv 0$, one has

$$
f(\omega)=\int_{0}^{\infty} \frac{d a}{a^{3}} \int_{\mathrm{SO}(3)} d \varrho W_{f}(\varrho, a)\left[A_{\psi}^{-1} R_{\varrho} D_{a} \psi\right](\omega) .
$$

Note again that the condition $\int_{0}^{2 \pi} d \varphi \psi(\theta, \varphi) \not \equiv 0$ is automatically satisfied for any nonzero axisymmetric (zonal) wavelet.

Correspondingly, instead of the familiar isometry property, one gets a Plancherel relation:

$$
\|f\|^{2}=\int_{0}^{\infty} \frac{d a}{a^{3}} \int_{\mathrm{SO}(3)} d \varrho W_{f}(\varrho, a) \overline{\widetilde{W}_{f}(\varrho, a)}
$$

where

$$
\widetilde{W}_{f}(\varrho, a):=\left\langle A_{\psi}^{-1} R_{\varrho} D_{a} \psi \mid f\right\rangle .
$$

The new fact here is the occurrence of the inverse $A_{\psi}^{-1}$ of the resolution operator in these formulas. This results from the square integrability of the representation (2.7) over the quotient space $X$, instead of the group itself.

\subsection{The geometrical or conformal method}

The group-theoretical method discussed so far yields a CWT on the sphere, but there is more. Indeed, there is a direct connection (unitary map) between the latter and the plane 2-D CWT, through the inverse stereographic projection, and it is uniquely specified by geometrical considerations. The result is twofold. ${ }^{40}$

(1) Uniqueness of the stereographic projection

Let $\mathrm{p}: S^{2} \backslash\{\mathrm{S}\} \rightarrow \mathbb{R}^{2}$ be a radial diffeomorphism, i.e., a $C^{\infty}$ bijection, from the 2 -sphere to the tangent plane at the North Pole:

$$
\mathrm{p}(\theta, \varphi)=(r(\theta), \varphi) \quad \text { with inverse } \mathrm{p}^{-1}(r, \varphi)=(\theta(r), \varphi) .
$$

Impose, in addition, that $\mathrm{p}$ be a conformal map, i.e., it preserves angles, or, equivalently, the metric $g^{\prime}$ induced by $\mathrm{p}$ on $\mathbb{R}^{2}$ is conformally equivalent to the Euclidean metric $g$ :

$$
g_{i j}^{\prime}(r, \varphi)=e^{\phi(r)} g_{i j}(r, \varphi), \quad i, j=1,2, \phi(r)>0 .
$$

Then one has uniquely $r(\theta)=2 \tan \frac{\theta}{2}$, i.e., $\mathrm{p}$ is the stereographic projection. 


\section{(2) Uniqueness of the stereographic dilation}

Let $D_{a}$ be a radial dilation on the sphere $S^{2}: D_{a}(\theta, \varphi)=\left(\theta_{a}(\theta), \varphi\right)$. Assume $D_{a}$ is a conformal diffeomorphism. Then one has uniquely $\tan \frac{\theta_{a}}{2}=a \tan \frac{\theta}{2}$, i.e., $D_{a}$ is the stereographic dilation (2.4).

The conclusion is that the two wavelet formalisms are equivalent. Let $\Pi$ : $L^{2}\left(S^{2}, d \mu\right) \rightarrow L^{2}\left(\mathbb{R}^{2}, d^{2} \vec{x}\right)$ be the unitary map induced by the stereographic projection:

$$
[\Pi F](\vec{x})=\frac{1}{1+(r / 2)^{2}} F\left(\mathrm{p}^{-1}(\vec{x})\right), \quad F \in L^{2}\left(S^{2}, d \mu\right)
$$

with inverse

$$
\left[\Pi^{-1} f\right](\theta, \varphi)=\frac{2}{1+\cos \theta} f(\mathrm{p}(\theta, \varphi)), \quad f \in L^{2}\left(\mathbb{R}^{2}, d^{2} \vec{x}\right)
$$

Then one obtains uniquely the spherical CWT from the plane (Euclidean) one, simply by transferring everything from the tangent plane to the sphere by inverse stereographic projection, the wavelets, the admissibility conditions, the directionality or steerability properties. ${ }^{40}$ For instance, every admissible Euclidean wavelet $\psi \in L^{2}\left(\mathbb{R}^{2}, d^{2} \vec{x}\right)$ yields an admissible spherical wavelet $\Pi^{-1} \psi \in L^{2}\left(S^{2}, d \mu\right)$. In particular, if $\psi$ is a directional wavelet, so is $\Pi^{-1} \psi$. An example is the spherical Morlet wavelet shown in Ref. 5, which has all the expected properties. More generally, any orthonormal, locally supported, wavelet basis in the plane (obtained by a multiresolution procedure) may be transferred and yields an analogous basis on the sphere. ${ }^{35}$

\subsection{Additional properties}

The spherical CWT just defined has further interesting properties, which are mostly described in our previous review paper. ${ }^{7}$

(1) Unlike the usual 2-D CWT, which is fully covariant with respect to translations, rotations and dilations, the spherical CWT is only partially covariant. It is covariant under motions on $S^{2}$ : for any $\varrho_{o} \in \mathrm{SO}(3)$, the transform of the rotated signal $f\left(\varrho_{o}^{-1} \omega\right)$ is the function $W_{f}\left(\varrho_{o}^{-1} \varrho, a\right)$. But it is not covariant under dilations. Indeed the wavelet transform of the dilated signal $\left(D_{a_{o}} f\right)(\omega)=\lambda\left(a_{o}, \theta\right)^{1 / 2} f\left(\omega_{1 / a_{o}}\right)$ is $\langle U(g) \psi \mid f\rangle$, with $g=a_{o}^{-1} \varrho a$, and the latter, while a well-defined element of $\operatorname{SO}_{o}(3,1)$, is not of the form $\sigma\left(\varrho^{\prime}, a^{\prime}\right)$. This reflects the fact that the parameter space $X$ of the spherical CWT is not a group, but only a homogeneous space.

(2) It yields an efficient analysis tool. Academic examples confirm that the spherical CWT behaves exactly as its plane counterpart, namely, it detects sharp boundaries on the sphere, independently of their position, even at the poles. And indeed it is currently applied by various groups for the analysis of the Cosmic Microwave Background radiation (CMB), in particular the question of the Gaussianity of its fluctuations. See Ref. 7 for further details and references to the original papers. 
(3) It has a correct Euclidean limit. By this we mean that, if we construct the transform on a sphere of radius $R$ and then let $R \rightarrow \infty$, the spherical CWT tends to the usual plane 2-D CWT on the tangent plane at the North Pole. We refer to Ref. 4 for mathematical details.

(4) Discretization of the spherical CWT leads to various types of (generalized) frames, either half-continuous (only the scale variable is discretized) or fully discrete. Technical details may be found in Ref. 9.

\section{The CWT on the two-sheeted hyperboloid}

\subsection{Geometry of the hyperboloid}

The two-sheeted hyperboloid $H^{2}$ is the dual manifold of the sphere $S^{2}$, with constant negative curvature and equation $x_{0}^{2}-x_{1}^{2}-x_{2}^{2}=1$. In polar coordinates, the upper sheet $H_{+}^{2}$ may be parameterized as $x=\left(x_{0}, x_{1}, x_{2}\right)=x(\chi, \varphi)$, where $x_{0}=\cosh \chi, x_{1}=\sinh \chi \cos \varphi, x_{2}=\sinh \chi \sin \varphi$ and $\chi \geqslant 0,0 \leqslant \varphi<2 \pi$ (Figure 2).

The motions on $H_{+}^{2}$ are of two types: (i) rotations : $x(\chi, \varphi) \mapsto\left(\chi, \varphi+\varphi_{0}\right)$; and (ii) hyperbolic motions : $x(\chi, \varphi) \mapsto\left(\chi+\chi_{0}, \varphi\right)$. Together they constitute the isometry group $\mathrm{SO}_{o}(2,1)$.

As in the case of the sphere $S^{2}$, the problem is how to define dilations on $H_{+}^{2}$. By definition, a dilation must be a homeomorphism $d_{a}: H_{+}^{2} \rightarrow H_{+}^{2}$ such that (i) $d_{a}$ monotonically dilates the azimuthal distance between two points, and (ii) the set $\left\{d_{a}, a>0\right\}$ is homomorphic to $\mathbb{R}_{*}^{+}: d_{a} d_{b}=d_{a b}, d_{a^{-1}}=d_{a}^{-1}, d_{1}=I$. The point is that here there are many possibilities. First, by comparison with the spherical case, it seems natural to define dilation through stereographic projection, say from the South pole to the equatorial plane $x_{0}=0$. As in the spherical case, one has a "pseudo-Iwasawa" decomposition:

$$
\mathrm{SO}_{o}(3,1)=\mathrm{SO}_{o}(2,1) \cdot \mathbb{R} \cdot N,
$$

where $\mathbb{R} \simeq \mathrm{SO}_{o}(1,1) \simeq\{$ boosts in the $z$-direction $\}$ and $N \simeq \mathbb{C}$. By the same tech-

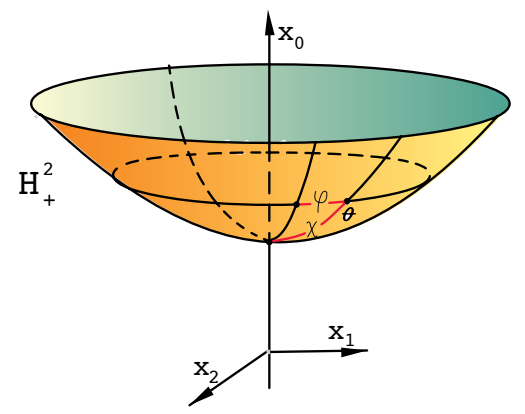

Fig. 2. The upper sheet of the two-sheeted hyperboloid. 


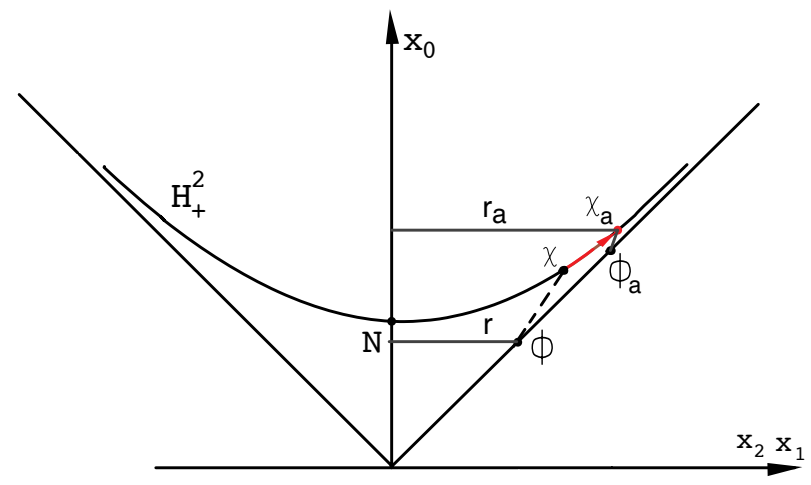

Fig. 3. Dilation through conic projection.

nique, one gets

$$
\tanh \frac{\chi_{a}}{2}=a \tanh \frac{\chi}{2}
$$

But this solution is problematic. Indeed, since $|\tanh \chi| \leqslant 1$, there is a critical value $\chi_{o}$ such that all points $\left(\chi_{o}, \varphi\right)$ will be sent to infinity by a finite dilation $a_{o}=\left(\tanh \chi_{o} / 2\right)^{-1}$. Moreover, for $a>a_{o}$, the dilation maps the upper sheet $H_{+}^{2}$ of the hyperboloid onto the lower sheet $H_{-}^{2}$. Indeed, the stereographic projection maps the upper sheet $H_{+}^{2}$ onto the interior of the unit disk in the equatorial plane and the lower sheet $H_{-}^{2}$ onto the exterior of the unit disk. ${ }^{3}$ Clearly this is unacceptable for setting up a CWT. In addition, there is no obvious representation of $\mathrm{SO}_{o}(3,1)$ in $L^{2}\left(H_{+}^{2}\right)$.

Therefore, other solutions have been proposed in Refs. 10, 11. We mention two of them.

(1) Dilation through conic projection:

The idea of this solution is to project the upper sheet of the hyperboloid $H_{+}^{2}$ onto its tangent half null-cone $\mathcal{C}_{+}^{2}$ :

$$
\mathcal{C}_{+}^{2}=\left\{x=\left(x_{0}, x_{1}, x_{2}\right) \in \mathbb{R}^{3}: x_{0}^{2}-x_{1}^{2}-x_{2}^{2}=0, x_{0}>0\right\} \text {, with dilation } x \mapsto a x .
$$

The conic projection $\Phi: H_{+}^{2} \rightarrow \mathcal{C}_{+}^{2}$ is given by

$$
\Phi(x)=2 \sinh \frac{\chi}{2} e^{i \varphi}, \quad \forall x=x(\chi, \varphi) \in H_{+}^{2} .
$$

The corresponding dilation is given by $\sinh \frac{\chi a}{2}=a \sinh \frac{\chi}{2}$ (Figure 3 ).

(2) Dilation through conic projection and "flattening":

For this solution, one projects $H_{+}^{2}$ onto the cone $\mathcal{C}_{+}^{2}$, and then vertically onto the plane $x_{0}=0$. The combined projection $\Pi_{1} \Phi: H_{+}^{2} \rightarrow \mathbb{R}^{2}$ is given by

$$
\Pi_{1} \Phi(x)=\sinh \chi e^{i \varphi}, \quad \forall x=x(\chi, \varphi) \in H_{+}^{2} .
$$


The corresponding dilation is given by $\sinh \chi_{a}=a \sinh \chi$.

Actually, there is a one-parameter family of possible projections $\Pi_{p} \Phi$ of this type,

$$
\Pi_{p} \Phi(x)=\frac{1}{p} \sinh p \chi e^{i \varphi}, \quad x=x(\chi, \varphi),
$$

leading to dilations $\sinh p \chi_{a}=a \sinh p \chi(p>0)$. Thus $p=\frac{1}{2}$ gives the dilation by conic projection, while $p=1$ yields the dilation by conic projection and flattening. However, the parameter $p$ does not seem to have a geometrical meaning.

\subsection{The CWT on the upper sheet of the hyperboloid}

In order to setup a CWT on the hyperboloid, the idea of Refs. 10, 11 is to exploit the existence of an appropriate integral transform on $L^{2}\left(H_{+}^{2}\right)$, the Fourier-Helgason transform, that defines harmonic analysis on $H^{2}$, including convolution theorems. ${ }^{23}$

The Fourier-Helgason transform is an isometry $\mathcal{F H}: L^{2}\left(H_{+}^{2}, d \mu\right) \rightarrow L^{2}(\mathcal{L}, d \eta)$, where $\mu$ is the $\mathrm{SO}_{o}(2,1)$-invariant measure on $H_{+}^{2}$ and $\mathcal{L}$ is a line-bundle over the space $\Xi=\mathbb{R}^{+} \times \mathbb{P} \mathcal{C}_{+}=\left\{(\nu, \xi): \nu \in \mathbb{R}^{+}, \xi \in \mathbb{P C _ { + }}\right\}$. Here $\mathbb{P C _ { + }}$ denotes the projective forward cone

$$
\mathbb{P C}_{+}=\left\{\xi \in \mathcal{C}_{+}^{2}: \lambda \xi \equiv \xi, \lambda>0, \xi_{0}>0\right\}
$$

and $L^{2}(\mathcal{L}, d \eta)$ is the Hilbert space of square integrable sections of $\mathcal{L}$, with an appropriate measure $d \eta(\nu, \xi) \cdot{ }^{10,11,23}$

Explicitly, the Fourier-Helgason (FH) transform and its inverse read, respectively,

$$
\begin{aligned}
\widehat{f}(\nu, \xi):= & \mathcal{F H}[f](\nu, \xi)=\int_{H_{+}^{2}} f(x)(x \cdot \xi)^{-\frac{1}{2}+i \nu} d \mu(x), \forall f \in C_{0}^{\infty}\left(H_{+}^{2}\right), \\
& \mathcal{F} \mathcal{H}^{-1}[g](x)=\int_{\Xi} g(\nu, \xi)(x \cdot \xi)^{-\frac{1}{2}-i \nu} d \eta(\nu, \xi), \forall g \in C_{0}^{\infty}(\mathcal{L}) .
\end{aligned}
$$

Then $\mathcal{F H}$ extends to an isometry of $L^{2}\left(H_{+}^{2}, d \mu\right)$ onto $L^{2}(\mathcal{L}, d \eta)$. In these relations, $(x \cdot \xi)^{-\frac{1}{2}-i \nu}$ is a hyperbolic plane wave, that is, an eigenfunction of the Laplacian over $H_{+}^{2}$, exactly as $\exp (i k \cdot x)$ is a Euclidean plane wave. We note that, if $f$ is rotation invariant, i.e., $\mathrm{SO}(2)$ invariant, then its $\mathrm{FH}$-transform $\widehat{f}(\nu, \xi)$ depends on $\nu$ only.

The second ingredient for designing a CWT on the hyperboloid is given by convolution theorems. First, given $f \in L^{2}\left(H_{+}^{2}\right)$ and $s \in L^{1}\left(H_{+}^{2}\right)$, their hyperbolic convolution is the following function on $\mathrm{SO}_{o}(2,1)$ :

$$
(f * s)(g)=\int_{H_{+}^{2}} f\left(g^{-1} x\right) s(x) d \mu(x) .
$$

Next, one may take the restriction to $H_{+}^{2}$, using a section [.] : $H_{+}^{2} \rightarrow \mathrm{SO}_{o}(2,1)$, and define

$$
(f * s)(y)=\int_{H_{+}^{2}} f\left([y]^{-1} x\right) s(x) d \mu(x), \quad y \in H_{+}^{2} .
$$


Then the key convolution theorem reads as follows.

Proposition 3.1. Given $f, s \in L^{2}\left(H_{+}^{2}\right)$ with $s$ rotation invariant, then $s * f \in$ $L^{1}\left(H_{+}^{2}\right)$ and

$$
\widehat{(s * f)}(\nu, \xi)=\widehat{f}(\nu, \xi) \widehat{s}(\nu) .
$$

We are now in a position to define the hyperbolic $C W T$, which actually looks exactly the same as its spherical counterpart.

Definition 3.2. Given an admissible hyperbolic wavelet $\psi$, the hyperbolic CWT of $f \in L^{2}\left(H_{+}^{2}\right)$ is

$$
\mathcal{W}_{f}(g, a):=\left\langle\psi_{a, g} \mid f\right\rangle=\int_{H_{+}^{2}} \overline{\psi_{a}\left(g^{-1} x\right)} f(x) d \mu(x), g \in \mathrm{SO}_{o}(2,1), a>0 .
$$

As in the spherical case, $\psi_{a}(x)=\lambda(a, x) \psi\left(d_{1 / a} x\right)$, with $d_{a}$ an appropriate dilation, and $\lambda(a, x)$ is the corresponding Radon-Nikodym derivative.

For instance, if $d_{a}$ is the dilation through conic projection, then $\lambda(a, x)=a^{-2}$. Note that, if the wavelet $\psi$ is axisymmetric, the hyperbolic CWT is a convolution:

$$
\mathcal{W}_{f}(g, a) \equiv \mathcal{W}_{f}(x, a)=\left(\overline{\psi_{a}} * f\right)(x), \text { where } g=[x], x \in H_{+}^{2} .
$$

It remains to state an appropriate wavelet admissibility condition.

Proposition 3.3. Let $\psi \in L^{1}\left(H_{+}^{2}\right)$ be axisymmetric and let $\alpha$ be a positive function on $\mathbb{R}_{*}^{+}$, for which there exists constants $m, M$ such that

$$
0<\mathrm{m} \leqslant \mathcal{A}_{\psi}(\nu):=\int_{0}^{\infty}\left|\widehat{\psi_{a}}(\nu)\right|^{2} \alpha(a) d a \leqslant \mathrm{M}<\infty .
$$

Then the linear operator $A_{\psi}$ on $L^{2}\left(H_{+}^{2}\right)$ defined by

$$
A_{\psi} f\left(x^{\prime}\right)=\int_{H_{+}^{2}} \int_{0}^{\infty} \mathcal{W}_{f}(x, a) \psi_{a,[x]}\left(x^{\prime}\right) \alpha(a) d a d x, x^{\prime} \in H_{+}^{2},
$$

is bounded with bounded inverse. The resolution operator $A_{\psi}$ is diagonal in FourierHelgason space (i.e., it is Fourier-Helgason multiplier):

$$
\widehat{A_{\psi} f}(\nu, \varphi)=\mathcal{A}_{\psi}(\nu) \widehat{f}(\nu, \varphi) .
$$

In other words, the family $\left\{\psi_{a,[x]}: a>0, x \in H_{+}^{2}\right\}$ is a continuous frame.

From Proposition 3.3, one obtains a reconstruction formula, which holds in the strong sense in $L^{2}\left(H_{+}^{2}\right)$ :

$$
f\left(x^{\prime}\right)=\int_{H_{+}^{2}} \int_{0}^{\infty} \mathcal{W}_{f}(x, a) A_{\psi}^{-1} \psi_{a,[x]}\left(x^{\prime}\right) \alpha(a) d a d x .
$$

As for the function $\alpha$, its choice is arbitrary, up to admissibility. For instance, if one chooses the dilation through conic projection and the function $\alpha(a)=a^{-\beta}, \beta>$ 


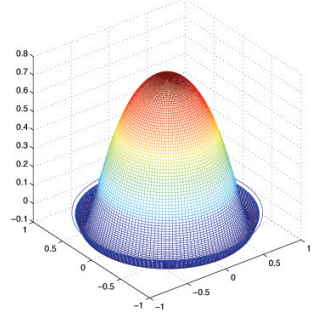

$a=1, \chi=0, \varphi=0$

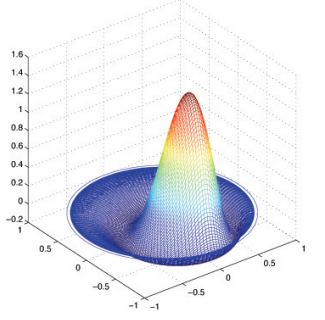

$a=0.5, \chi=1, \varphi=\pi / 2$

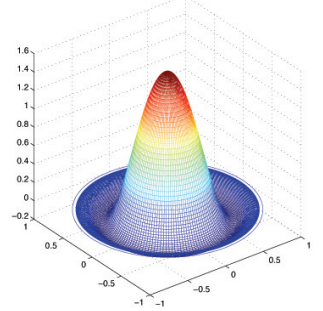

$a=0.5, \chi=0, \varphi=0$

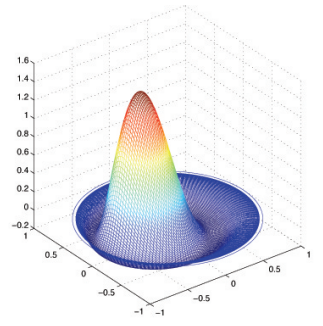

$a=0.5, \chi=0.75, \varphi=\pi$

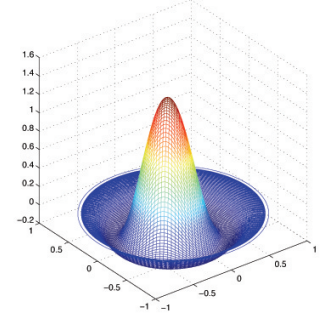

$a=0.5, \chi=1, \varphi=3 \pi / 4$

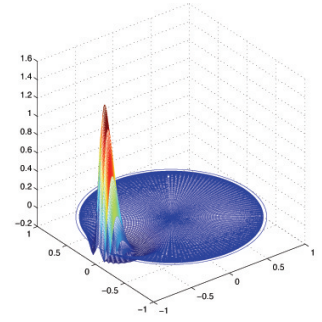

$a=0.5, \chi=2.75, \varphi=3 \pi / 4$

Fig. 4. The hyperbolic DOG wavelet at different scales and positions.

0 , then an axisymmetric wavelet $\psi \in L^{2}\left(H_{+}^{2}\right)$ is admissible either if $1<\beta \leqslant 2$ and $\psi$ is integrable, or $\beta>2$ and the following zero-mean condition is satisfied:

$$
\int_{H_{+}^{2}} \psi(x) d \mu(x)=0, \quad x=(\chi, \varphi) .
$$

Exactly as in the spherical case, a typical hyperbolic wavelet is the hyperbolic DOG at scale $a$ (which is axisymmetric):

$$
\psi_{\text {HDOG }}(\chi, \varphi)=\frac{1}{a} \exp \left[-\frac{1}{a^{2}} \sinh ^{2}\left(\frac{\chi}{2}\right)\right]-\frac{1}{4 a} \exp \left[-\frac{1}{4 a^{2}} \sinh ^{2}\left(\frac{\chi}{2}\right)\right]
$$

(here one uses again the dilation via conic projection). This wavelet is presented in Figure 4 at two scales $(a=1, a=0.5)$ and various positions $(\chi, \varphi)$ on $H_{+}^{2}$. This allows to visualize the action of the various operators upon the wavelet (dilation, hyperbolic translation, rotation). Note that, for better readability, the visualization is made in the open unit disk obtained from $H_{+}^{2}$ by stereographic projection. ${ }^{10}$

Finally we notice that the Euclidean limit also exists in the hyperbolic case. Taking a hyperboloid $H_{R}^{2}$ of radius $R$, that is, of equation $x_{0}^{2}-x_{1}^{2}-x_{2}^{2}=R^{2}$, and letting $R \rightarrow \infty$, one shows that the Fourier-Helgason transform on $H_{+R}^{2}$ tends to the usual Fourier transform in the plane, mirroring the group contraction $\mathrm{SO}(2,1) \rightarrow$ $\mathrm{E}(2)$, the 2-D Euclidean group (upon adding dilations, each of the two groups gives the parameter space of the corresponding CWT). ${ }^{10}$ Then, taking once again the 
dilation via conic projection, so that $\lambda(a, x)=a^{-2}$, one shows that the admissibility condition contained in (3.4) goes into the corresponding admissibility condition of the usual 2-D plane wavelets, namely $\int_{\mathbb{R}^{2}}|\widehat{\psi}(\vec{k})||\vec{k}|^{-2} d^{2} \vec{k}<\infty$. As a consequence, the necessary zero-mean admissibility condition (3.5) goes into the corresponding zero-mean condition of the usual 2-D plane wavelets $\int_{\mathbb{R}^{2}} \psi(\vec{x}) d^{2} \vec{x}=0$.

\section{The CWT on other conic sections}

\subsection{The case of the paraboloid}

The (axisymmetric) paraboloid is the manifold of equation $P^{2}=\left\{\left(x_{0}, x_{1}, x_{2}\right) \in \mathbb{R}^{3}\right.$ : $\left.x_{0}=x_{1}^{2}+x_{2}^{2}\right\}$. This is a singular limit case between the sphere $S^{2}$ and the twosheeted hyperboloid $H^{2}$. The paraboloid $P^{2}$ does not have a constant curvature and it also lacks a large isometry group, so that the general coherent state formalism cannot be used. Therefore, designing a CWT on $P^{2}$, by a limiting procedure or otherwise, is bound to be a difficult process.

A different approach has been suggested, however., ${ }^{2,27}$ The idea is to consider instead of $P^{2}$ a related manifold, namely, the paraboloid with apex removed $\mathfrak{P}=$ $P^{2} \backslash\{(0,0,0)\}$. Then one notes that the set $\mathrm{P}$ of $3 \times 3$ matrices of the form $g=$ $\operatorname{diag}\left(a^{2}, a r_{\theta}\right)$, whith $a>0, r_{\theta} \in \mathrm{SO}(2)$, leaves both $P^{2}$ and $\mathfrak{P}$ invariant. Next one embeds $\mathrm{P}$ into the group

$$
\mathrm{G}_{\mathfrak{P}}=\left\{g(\vec{b}, a, \theta):=\left(\begin{array}{cc}
a^{2} & \overrightarrow{0}^{T} \\
\vec{b} & a r_{\theta}
\end{array}\right): a>0, \vec{b} \in \mathbb{R}^{2}, 0 \leqslant \theta<2 \pi\right\} .
$$

This group $\mathrm{G}_{\mathfrak{P}}$ is a Lie group, nonunimodular, similar to, but different from $\operatorname{SIM}(2)$. It turns out that this group factorizes as $\mathrm{G}_{\mathfrak{P}}=P H$, where $H$ acts as a shear group on $\mathbb{R}^{3}$. Then $P \simeq \mathrm{G}_{\mathfrak{P}} / H$ and is homeomorphic to $\mathrm{G}_{\mathfrak{P}}$. Thus $\mathrm{P}$ has a natural action on $\mathfrak{P}$.

Furthermore, the group $\mathrm{G}_{\mathfrak{P}}$ has an irreducible unitary representation $U$ in $L^{2}\left(\mathrm{G}_{\mathfrak{P}}, d \mu_{\mathfrak{P}}\right)$, where the measure $d \mu_{\mathfrak{P}}$ is invariant under the action of $P$. This representation is square integrable, so that the general coherent state formalism may be applied, as in the spherical case. The outcome is a time-frequency transform, which acts on $\mathfrak{P}$ much as a CWT in $\vec{k}$-space (momentum space).

However, this transform does not involve dilations, so it is not really a wavelet transform. Then it is suggested to design first a CWT on a circular cylinder and then transport it to $\mathfrak{P}$ by a homeomorphism. But the resulting CWT has other drawbacks, so that the problem remains unsolved, in our opinion. We will come back to this case elsewhere.

\subsection{The CWT on general conical sections}

According to Apollonius, ${ }^{42}$ the conic sections are the sphere $S^{2}$, the paraboloid $P^{2}$ and the two-sheeted hyperboloid $H^{2}$. All three are obtained as sections of a double 
null-cone

$$
\mathcal{C}_{0}^{3}=\left\{\left(x_{0}, x_{1}, x_{2}, x_{3}\right) \in \mathbb{R}^{4}: x_{0}^{2}-x_{1}^{2}-x_{2}^{2}-x_{3}^{2}=0\right\}
$$

by a plane $x_{0}=1+\tan \alpha\left(x_{3}-2\right), 0 \leqslant \alpha \leqslant \pi$ (hence the name). Thus one gets the full family of conic sections, indexed by the parameter $\alpha$ :

. For $\alpha=0$, the unit sphere $S^{2}$;

. For $0<\alpha<\pi / 4$, an ellipsoid;

. For $\alpha=\pi / 4$, the paraboloid $P^{2}$;

. For $\pi / 4<\alpha<\pi / 2$, a two-sheeted hyperboloid;

. For $\alpha=\pi / 2$, the two-sheeted hyperboloid $H^{2}$.

As usual, the key is to define appropriate dilations. A possible technique consists in introducing generalized projective coordinates. On the section indexed by the tilt angle $\alpha$, one puts:

$$
u_{i}=\frac{1-2 \tan \alpha}{x_{0}-x_{3} \tan \alpha} x_{i}, i=1,2,3 .
$$

For the sphere $S^{2}$, corresponding to $\alpha=0$, this gives the familiar projective coordinates $u_{i}=x_{i} / x_{0}$. Then the dilation is obtained by a Lorentz boost of parameter $t \in \mathbb{R}$ along the axes $x_{0}, x_{3}$, followed by a projection back to the section via the projective coordinates (4.1). Applying this method to the sphere $S^{2}$, one recovers exactly the stereographic dilation $\tan \frac{\theta_{a}}{2}=a \tan \frac{\theta}{2}$, with $a=e^{t}$.

The technique is illustrated in Figure 5 for the case of an ellipsoidal section. The section is represented by the ellipse, with S, N representing the South, resp. North, pole of the sphere or the ellipsoid. Then one proceeds in two steps: (i) a boost $P \rightarrow P^{\prime}$; (ii) a projection back to the section by homogeneous coordinates $P^{\prime} \rightarrow \Pi\left(P^{\prime}\right)$. An alternative way consists in making first the boost $P \rightarrow P^{\prime}$, as above, then coming back to the section by another Lorentz boost in the plane $P^{\prime} O x_{0}$.

However, applying the same procedure in the case of the hyperboloid $H^{2}$, one recovers in both cases the stereographic dilation, which mixes the two sheets and is therefore unacceptable.

One may also consider an alternative way of generating sections, this one purely group-theoretical. The idea is simply to start from the standard sections $S^{2}$ or $H^{2}$ and generate new sections by a boost $x \mapsto x^{\prime}$ of parameter $\chi$ along the axes $x_{0}, x_{2}$. From $S^{2}$, with equation $x_{0}=1$, one gets in this way an ellipsoid of equation

$$
x_{1}^{\prime 2}+\left(\frac{x_{0}^{\prime}-\cosh \chi}{\sinh \chi}\right)^{2}+x_{3}^{\prime 2}=x_{1}^{\prime 2}+\left(\frac{x_{2}^{\prime}-\sinh \chi}{\cosh \chi}\right)^{2}+x_{3}^{\prime 2}=1 .
$$

This is indeed the equation of an ellipsoid of revolution around the $x_{0}$ axis, of eccentricity $1 / \cosh ^{2} \chi$ in the $\left(x_{1}, x_{2}\right)$ plane and $1 / \sinh ^{2} \chi$ in the $\left(x_{1}, x_{0}\right)$ plane.

From $H^{2}$, with equation $x_{3}=1$, one gets a two-sheeted hyperboloid. In this approach, it should be noted that the paraboloid, as limit from both sides, becomes a degenerate half-line (see Figure 6). 


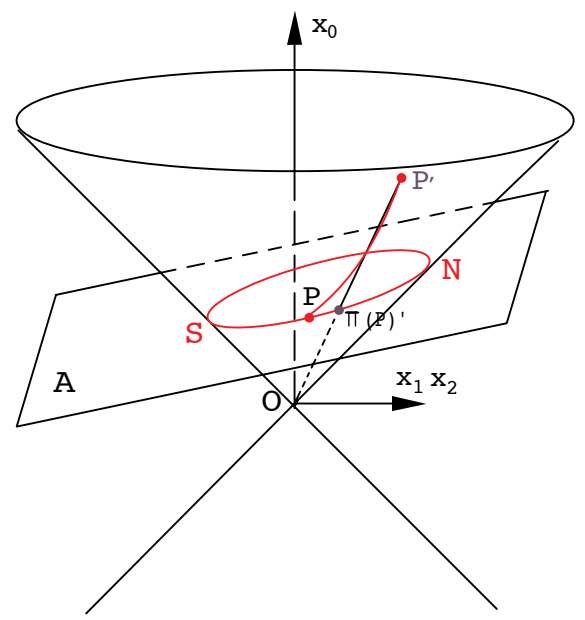

Fig. 5. Dilation on an ellipsoidal section via projective coordinates.
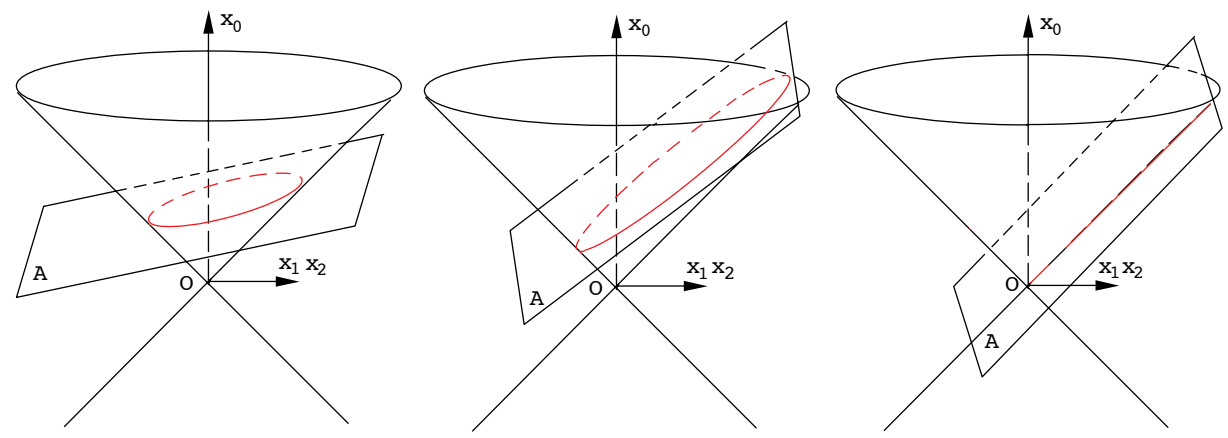

Fig. 6. Generation of conic sections.

In all cases, the strategy for building a CWT on an ellipsoidal section is the following. (i) Start with the spherical section that gives $S^{2}$ and consider the usual representation $U$ of the Lorentz group $\mathrm{SO}_{o}(1,3)$ in $L^{2}\left(S^{2}\right)$; (ii) Any other smooth section $\sigma\left(S^{2}\right)$ of the same type allows to bring the action of $\mathrm{SO}_{o}(1,3)$ to $\sigma\left(S^{2}\right)$ and induces an isometry $V_{\sigma}: L^{2}\left(S^{2}\right) \rightarrow L^{2}\left(\sigma\left(S^{2}\right)\right.$ ); (iii) One gets a new UIR of $\mathrm{SO}_{o}(1,3)$ in $L^{2}\left(\sigma\left(S^{2}\right)\right)$ by $V_{\sigma} \circ U \circ V_{\sigma}^{-1}$. Then the construction of wavelets on the new section is immediate.

\section{Outcome}

Mathematical aestheticism shouldn't lead us to forget that one of the key ingredients in the success of wavelets is the very efficient algorithmic structure that sustains them. Although developing fast algorithms is out of the scope of this paper, it 
can be enlightening to point at general arguments that lead, or may lead, to such algorithms. In the particular case of the Continuous Wavelet Transform in $\mathbb{R}^{d}$, everything is rather simple: at fixed scale and rotation angle, the CWT is indeed a convolution and can be sampled and efficiently computed using the FFT algorithm. The same is true for the CWT on the sphere, but with an extra twist: the transform is a correlation that, at each scale, maps functions on $S^{2}$ to functions on the rotation group $S O(3)$. There again, fast Fourier transform algorithms have been used to compute the CWT at reduced cost (see for example Refs. 20, 21, 41). Difficulties increase event further in the case of the CWT on the hyperboloid. Though the transform is once again of convolution type and diagonalized in the Fourier domain, implementation of the associated Fourier transform (the Helgason transform) hasn't been fully studied despite early interest. ${ }^{28}$ Finally, as for the case of general conic sections, everything has to be invented. Note however that the section mechanism using the isometry $V_{\sigma}$ above suggests performing most of the computation on $S^{2}$ and then mapping the result to the target surface. In that case, most of the algorithmic structure is similar to the well-controlled case of the CWT on the sphere.

The general conclusion is that wavelets on conic sections are an active field of research, that keeps finding exciting applications, essentially in astrophysics and in optics (catadioptric image processing). Progress is also made on the mathematical side, but much work remains to be done, especially on the nonspherical cases. Actually other non-Euclidean manifolds may also be considered, but this another story.

\section{References}

1. S.T. Ali, J-P. Antoine, and J-P. Gazeau, Coherent States, Wavelets and Their Generalizations, Springer-Verlag, New York, Berlin, Heidelberg, 2000

2. S.T. Ali and G. Honnouvo, private communication.

3. J-P. Antoine and P. Vandergheynst, Wavelets on the $n$-sphere and other manifolds, J. Math. Phys. 39 (1998), 3987-4008.

4. J-P. Antoine and P. Vandergheynst. Wavelets on the 2-sphere: A group-theoretical approach, Applied Comput. Harmon. Anal. 7 (1999), 262-291.

5. J-P. Antoine, L. Demanet, L. Jacques, and P. Vandergheynst, Wavelets on the sphere: Implementation and approximations, Applied Comput. Harmon. Anal. 13 (2002), 177200.

6. J-P. Antoine, R. Murenzi, P. Vandergheynst, and S.T. Ali, Two-dimensional Wavelets and Their Relatives, Cambridge University Press, Cambridge (UK), 2004.

7. J-P. Antoine and P. Vandergheynst, Wavelets on the two-sphere and other conic sections, J. of Fourier Analysis and Appl. 13 (3) (2007) to appear.

8. A.O. Barut and R. Rączka, Theory of Group Representations and Applications, PWN, Warszawa, 1977.

9. I. Bogdanova, P. Vandergheynst, J-P. Antoine, L. Jacques, and M. Morvidone, Stereographic wavelet frames on the sphere, Applied Comput. Harmon. Anal. 26 (2005), 223-252.

10. I. Bogdanova, Wavelets on non-Euclidean manifolds, PhD thesis, EPFL, 2005.

11. I. Bogdanova, P. Vandergheynst, and J-P. Gazeau, Continuous wavelet transform on the hyperboloid, preprint, EPFL (2005). Applied Comput. Harmon. Anal., submitted. 
12. I. Bogdanova, X. Bresson, J-Ph. Thiran, and P. Vandergheynst, Scale space analysis and active contours for omnidirectional images, preprint EPFL (2006); IEEE Trans. Image Proc., submitted.

13. T. Bülow, Multiscale image processing on the sphere, in DAGM-Symposium, (2002) pp. 609-617.

14. A. Chambodut, I. Panet, M. Mandea, M. Diament, M. Holschneider, and O. Jamet, Wavelet frames: an alternative to spherical harmonic representation of potential fields, Geophys. J. Internat. 163 (2005), 875-899.

15. W. Freeden and U. Windheuser, Combined spherical harmonic and wavelet expansion - A future concept in Earth's gravitational determination, Applied Comput. Harmon. Anal. 4, (1997) 1-37.

16. W. Freeden, T. Gervens, and M. Schreiner, Constructive Approximation on the Sphere, with Applications to Geomathematics, Clarendon Press, Oxford, 1997.

17. W. Freeden, T. Maier, and S. Zimmermann, A survey on wavelet methods for (geo)applications, Revista Mathematica Complutense 16, (2003), 277-310.

18. C. Geyer and K. Daniilidis, Catadioptric projective geometry, Int. J. Computer Vision 45 (2001), 223-243.

19. R. Gilmore, Lie Groups, Lie Algebras, and Some of Their Applications, Wiley, New York and London, 1974.

20. K.M. Grski, E. Hivon, A.J. Banday, B.D. Wandelt, F.K. Hansen, M. Reinecke, and M. Bartelmann, HEALPIX - a Framework for High Resolution Discretization, and Fast Analysis of Data Distributed on the Sphere, Astrophys. J. 622 (2005), 759-771.

21. D.M. Healy, Jr., P. Kostelec, D. Rockmore, and S. Moore, FFTs for the 2-SphereImprovements and Variations, J. Fourier Anal. Appl. 9 (4) (2003), 341-385.

22. S. Helgason, Differential Geometry, Lie Groups, and Symmetric Spaces, Academic Press, New York, 1978, and Amer. Math. Soc., Providence, RI, 2001.

23. S. Helgason, Geometric Analysis on Symmetric Spaces, Amer. Math. Soc., Providence, RI, 1994.

24. M. Holschneider, Continuous wavelet transforms on the sphere, J. Math. Phys. 37 (1996), 4156-4165.

25. M. Holschneider, A. Chambodut A, and M. Mandea, From global to regional analysis of the magnetic field on the sphere using wavelet frames, Phys. Earth Plan. Inter. 135 (2003), 107-124.

26. M. Holschneider, Poisson wavelets on the sphere, J. Fourier Anal. Appl. 13 (2007), to appear.

27. G. Honnouvo, Gabor Analysis and Wavelet Transforms on some Non-Euclidean 2Dimensional Manifolds, PhD Thesis, Concordia Univ., Montreal, PQ, Canada, 2007.

28. P. Kuchment, Generalized transforms of Radon type and their applications, in G. Olafsson and E.T. Quinto (eds.), The Radon Transform, Inverse Problems, and Tomography, Proc. Symp. Appl. Math. v. 63, AMS, Providence, RI, 2006, pp.67 - 91.

29. A. Kunoth and J. Sahner, Wavelets on manifolds: An optimized construction, Math. Comput. 75 (255) (2006), 1319-1349.

30. H.N. Mhaskar, F.J. Narcowich, J. Prestin, and J.D. Ward, Polynomial frames on the sphere, Adv. Comput. Math., 13 (2000) 387-403.

31. D. Potts, G. Steidl, and M. Tasche, Kernels of spherical harmonics and spherical frames, in Advanced Topics in Multivariate Approximation, pp. 287-301; F. Fontanella, K. Jetter and P.J. Laurent (eds.), World Scientific, Singapore, 1996.

32. D. Roşca, Locally supported rational spline wavelets on the sphere, Math. Comput. 74 (252) (2005), 1803-1829.

33. D. Roşca, Piecewise constant wavelets defined on closed surfaces, J. Comput. Anal. 
Appl. 8 (2) (2006), 121-132.

34. D. Roşca, Wavelet bases on the sphere obtained by radial projection, J. Fourier Anal. Appl. 13 (3) (2007) to appear.

35. D. Roşca and J-P. Antoine, Locally supported orthogonal wavelet bases on the sphere via stereographic projection, in preparation

36. Schröder, P. and Sweldens, W. (1995). Spherical wavelets: Efficiently representing functions on the sphere, in Computer Graphics Proceedings (SIGGRAPH1995), SIGGRAPH, ACM, pp. 161-172.

37. R. Takahashi, Sur les représentations unitaires des groupes de Lorentz généralisés, Bull. Soc. Math. France 91 (1963), 289-433.

38. B. Torrésani, Position-frequency analysis for signals defined on spheres, Signal Proc. 43 (2005), 341-346.

39. I. Weinreich, A construction of $C^{1}$-wavelets on the two-dimensional sphere, Applied Comput. Harmon. Anal. 10 (2001), 1-26.

40. Y. Wiaux, L. Jacques, and P. Vandergheynst, Correspondence principle between spherical and Euclidean wavelets, Astrophys. J. 632 (2005), 15-28.

41. Y. Wiaux, L. Jacques, and P. Vandergheynst, Fast directional correlation on the sphere with steerable filters, Astrophys. J., 652 (2006), 820-832.

42. See Wikipedia, http://en.wikipedia.org/wiki/Apollonius_of_Perga 